$\begin{array}{llllllllll}\text { A C T A } & \text { C H E M C A } & \text { S C A N D I N A V I C A } & 27 & \text { (1973) } & 985-989\end{array}$

\title{
Effect of 1-Hydroxymethyl- and 1-Methyl-1-aminoalkanes on Enzymatic Leucine-2-oxoglutarate Transamination
}

\author{
RAIMO RAUNIO, TIMO KORPELA and ANTTI MUSTRANTA
}

Department of Biochemistry, University of Turku, SF-20500 Turku 50, Finland

\begin{abstract}
Studies were made on the effect of some 1-hydroxymethyl- and 1-methyl-1-aminoalkanes on the enzymatic leucine-2-oxoglutarate transamination reaction catalyzed by Escherichia coli cell extract, and on the transamination of the analogs and their effect on the growth of $E$. coli. All the analogs inhibited the enzymatic leucine-2-oxoglutarate transamination reaction and this inhibition was competitive with leucine. The analogs were not able to donate an amino group to 2-oxoglutarate when $E$. coli cell extract was used as enzyme source and they acted as growth inhibitors when $E$. coli cells were cultured in a simple glucose-mineral salt medium. It is concluded that the carboxylate group of leucine is essential for the enzymatic leucine-2oxoglutarate transamination reaction.
\end{abstract}

In the transamination reaction between an amino acid and 2-oxoglutarate, 1 the 2-amino group and $\alpha$-carbon hydrogen play the key role in the formation of a Schiff base with amino acid and pyridoxal-5' - phosphate and in the formation of the end product, a keto acid. The role of the carboxyl group of the amino acid in the reaction is poorly understood. There is some experimental evidence to suggest that the carboxylate anion is involved in the reaction and it is supposed that this anion becomes attached to the apoenzyme part, effecting the breakage of a bond specific to the reaction. ${ }^{1-3}$

We selected some 1-hydroxymethyl- and 1-methylalkylamines and tested their effect on the enzymatic leucine-2-oxoglutarate transamination reaction and their ability to donate an amino group to 2-oxoglutarate catalyzed by partially purified $E$. coli leucine aminotransferase (EC 2.6.1.6).

\section{MATERIALS AND METHODS}

Organism. A wild-type Escherichia coli U5-41 was used in the growth inhibition studies and as source for leucine aminotransferase. The compositions of the transfer, inoculation and test media have been reported earlier. ${ }^{4}$

Assay methods. Chromatographic and isotopic methods were used in the leucine aminotransferase activity determinations. The isotopic method, in which the reaction

Acta Chem. Scand. 27 (1973) No. 3 
mixture contained $5 \mathrm{mM}$ L-leucine, $25 \mathrm{mM} 2$-oxoglutarate and $10 \mu \mathrm{M}$ pyridoxal-5' phosphate in $50 \mathrm{mM}$ Tris-HCl buffer, $\mathrm{pH} 8.0$, has been published elsewhere. ${ }^{5}$ Analogs were added to the reaction mixture to give 10 or $30 \mathrm{mM}$ concentrations. In this assay $0.05-0.1$ $\mu \mathrm{Ci}$ of uniformly labeled $\mathrm{I}_{-}$-leucine-U-C-14 (specific activity $344 \mathrm{mCi} / \mathrm{mmol}$ ) was added to the reaction mixture to the volume of $1 \mathrm{ml}$. In some experiments lower leucine concentrations were used to obtain higher counts and these experiments are mentioned later. The radiocarbon was counted in a scintillation solution containing $3.92 \mathrm{~g}$ of PPO (2,5diphenyloxazole) and $0.08 \mathrm{~g}$ of bis-MSB ( $p$-bis(o-methylstyryl)benzene) in 11 of toluene. The liquid scintillation counter was Decem NTL ${ }^{314}$ from Wallac Co., Turku, Finland.

The chromatographic method was a modification of a previously published method in which glutamate formed from 2-oxoglutarate was estimated after it had been separated by paper chromatography and sprayed with ninhydrin. The modification consisted of using lower substrate concentrations, as in the isotopic assay.

Enzyme preparation. Leucine aminotransferase (EC 2.6.1.6) was partially purified from a wild-type $E$. coli U5-41 by a method presented earlier."

Analogs and their preparation. The following 2-amino compounds were purchased as follows: 2-aminoethanol, DL-2-aminobutyric acid from British Drug Houses Ltd, Poole, England, 2-aminopropanol, 2-aminobutanol, 2-aminopropane, 2-aminobutane, 2-amino4-methylhexane from Koch-Light Laboratories Ltd, Colnbrook, England, 2-aminoethane, 2-amino-3-methylpentane, 2-amino-6-methylheptane from Fluka AG, Buchs, Switzerland, 2-aminopentane and 2-aminohexane from K \& K Laboratories Inc., Hollywood, California. Leucinol, isoleucinol, valinol, norleucinol, and norvalinol were prepared from the corresponding L-amino acids by the method of Vogl and Pöhm. ${ }^{6}$ An ascending paper chromatographic method, with butanol-acetic acid-water $4: 1: 1(\mathrm{v} / \mathrm{v} / \mathrm{v})$ as solvent, was used to test that the preparations were free from amino acids, and the absence of a carboxylic group and the presence of the amino group were demonstrated by infrared analysis. All other reagents were chromatographically pure reagent grade compounds.

NMR spectra. The differences between chemical shifts were determined on a $60 \mathrm{MHz}$ Perkin-Elmer R 10 NMR spectroscope at $33.5^{\circ}$.

Table 1. Effect of 1-hydroxymethyl- and 1-methyl-1-alkylamines on the enzymatic leucine-2-oxoglutarate transamination catalyzed by partially purified leucine aminotransferase from $E$. coli. Initial velocities were followed in the reaction mixture containing $5 \mu \mathrm{mol}$ L-leucine, $3.4 \times 10^{-4} \mu \mathrm{mol}$ uniformly labelled $\mathrm{L}_{\text {-leucine }}(0.1 \mu \mathrm{Ci}), 25 \mu \mathrm{mol} 2$-oxoglutarate, $0.01 \mu \mathrm{mol}$ pyridoxal-5-phosphate in $50 \mathrm{mM}$ Tris-HCl buffer, pH 8.0, plus 10 $\mathrm{mM}$ or $30 \mathrm{mM}$ analog. The total reaction volume was $1.0 \mathrm{ml}$ and the activities are compared to the activity without analog which is taken as 100 .

\begin{tabular}{lrr}
\multicolumn{1}{c}{ Analog } & \multicolumn{2}{c}{ Concentration } \\
& $10 \mathrm{mM}$ & $30 \mathrm{mM}$ \\
\hline None & 100 & 100 \\
2-Aminoethanol & 91 & 70 \\
2-Aminopropanol & 85 & 77 \\
2-Aminobutanol & 96 & 71 \\
Norvalinol & 80 & - \\
Norleucinol & 91 & 84 \\
Valinol & 60 & - \\
Leucinol & 90 & 70 \\
Isoleucinol & 89 & 72 \\
2-Aminoethane & 76 & 33 \\
2-Aminopropane & 84 & 30 \\
2-Aminobutane & 62 & 25 \\
2-Aminopentane & 57 & 40 \\
2-Aminohexane & 67 & 25 \\
2-Amino-3-methylpentane & 59 & 36 \\
2-Amino-4-methylhexane & 74 & 37 \\
2-Amino-6-methylheptane & 78 & 26 \\
I-Isoleucine & 51 & 19 \\
\hline
\end{tabular}




\section{RESULTS}

Effect of analogs on the enzymatic transamination of leucine. When one of the analogs at a concentration of $10 \mathrm{mM}$ or $30 \mathrm{mM}$ was added to the reaction mixture containing $5 \mathrm{mM} \mathrm{L}$-leucine and $25 \mathrm{mM}$ 2-oxoglutarate, the reaction rate was lower than in its absence. The results are tabulated in Table 1.

With all the 1-hydroxymethyl- and 1-methyl type analogs a sixfold amount of the analog, as compared with the leucine concentration, caused marked inhibition. The inhibition was higher with 1-methyl analogs than with 1-hydroxymethyl compounds. No marked differences were found with different side chains of alkylamines.

Inhibition constants and type of inhibition. Fig. 1 shows the graphical determination of inhibitor constants $K_{\mathrm{i}}$ and they were $2.0 \mathrm{mM}$ for leucinol, 1.7 $\mathrm{mM}$ for 2-amino-4-methylhexane and $0.6 \mathrm{mM}$ for isoleucine. The Dixon plot method was used to determine the constants, and the activity assays were made with the isotopic method. ${ }^{7}$ The type of inhibition was competitive in
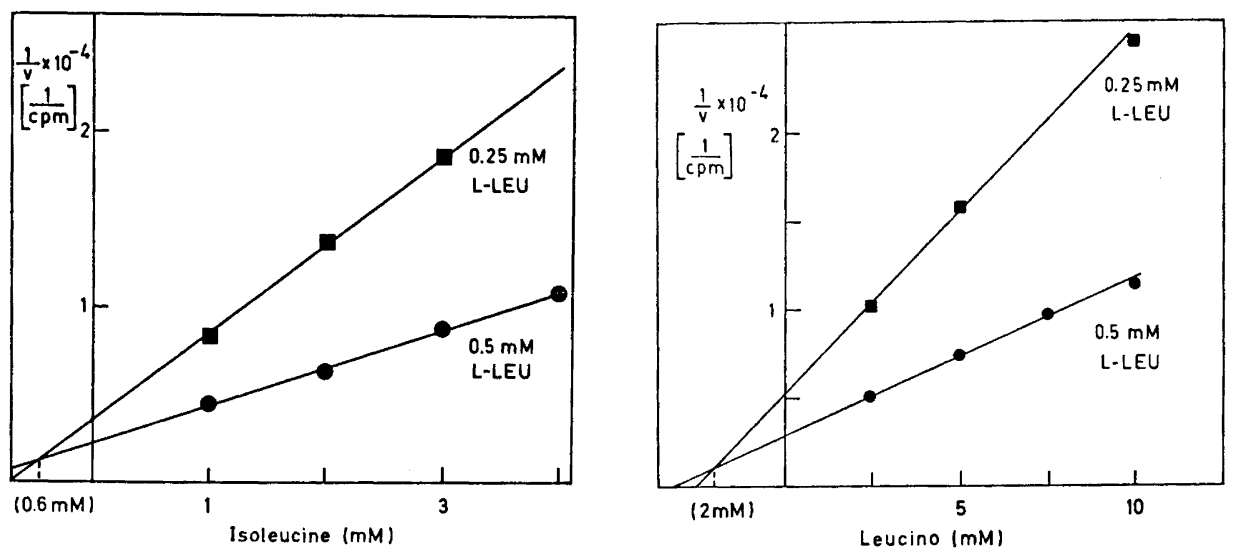

Fig. 1. Estimation of the inhibition constants for leucine-2-oxoglutarate transamination inhibitors, isoleucine, leucinol, and 2-amino-4-methylhexane, using Dixon's plot. The reaction mixture contained 0.25 or $0.5 \mathrm{mM} \mathrm{L}$-leucine. The isotopic method was used in the assay.

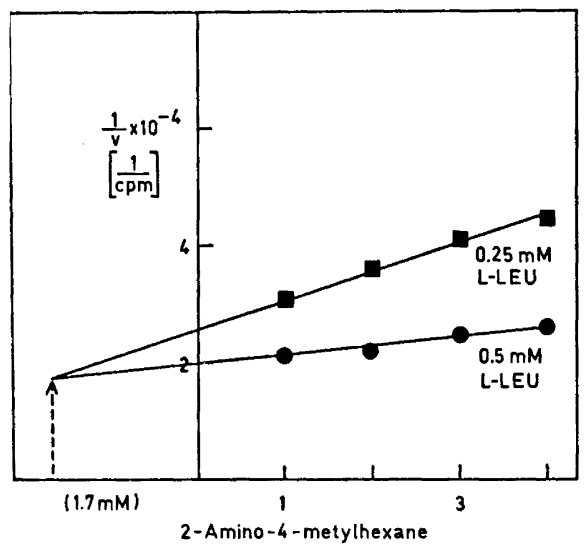


all three cases studied. The $K_{m}$ value for leucine was $0.3 \mathrm{mM}$ with $E$. coli U541 enzyme.

Transamination of analogs. The deamination of the analogs used was studied by following the release of hydrogen from the $\alpha$-carbon in the presence of 2-oxoglutarate by the NMR technique and also the formation of glutamate from 2-oxoglutarate. Fig. 2 shows the spectra obtained by the NMR technique when the reaction mixture contained $L_{4}$-leucine and isoleucinol as amino donor.
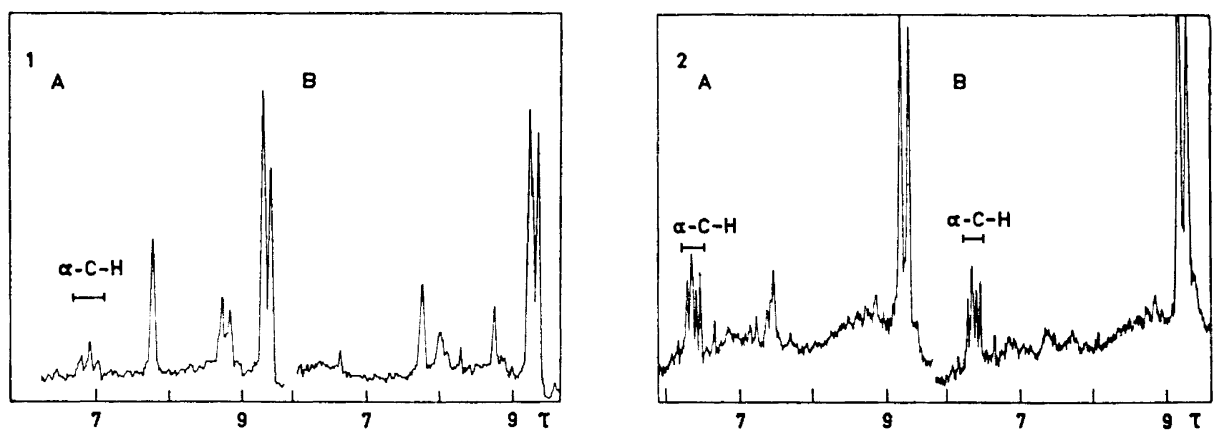

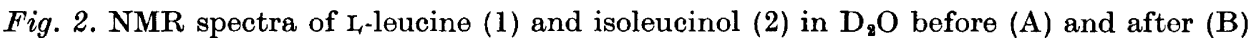
incubation of $30 \mathrm{mg}$ of amino compound for $24 \mathrm{~h}$ at $37^{\circ}$ in the presence of $30 \mathrm{mg} 2$-oxoglutarate and $30 \mathrm{mg}$ of $E$. coli protein containing leucine aminotransferase per $\mathrm{ml}$ (pD was 8.0 and it was adjusted with $\mathrm{NaOD}$ ). The protein was precipitated by heating and centrifuged off before NMR analysis. The position of the $\alpha$-carbon hydrogen is marked as $\alpha-\mathrm{C}-\mathrm{H}$. There was no release of hydrogen from the other analogs mentioned in Table 1.

The results showed that none of the analogs, except leucinol with an activity less than $10 \%$ of that of leucine, acted as an amino donor to keto acid or was able to release hydrogen from the $\alpha$-carbon. The concentration of the amino acid or amino compound in the reaction mixture was $30 \mathrm{mM}$ in the chromatographic method, in which the formation of glutamate was followed. The composition of the reaction mixture in the NMR analysis is mentioned in Fig. 2.

Inhibition of $E$. coli growth by analogs. The effects of the analogs on the growth of a wild-type, branched-chain amino acid resistant Escherichia coli U5-41 were also studied. The additions were made at concentrations of $10^{-4}$ to $10^{-1} \mathrm{M}$ to the medium, which contained glucose, ammonium chloride, and mineral salts. 5 The analogs were added to the medium aseptically before drop inoculation of $10 \mathrm{ml}$ of the medium. The tubes were incubated overnight at $37^{\circ}$ without shaking and the turbidities were determined with a Klett colorimeter, using filter No. $42(400-450 \mathrm{~nm})$. The $50 \%$ inhibition concentrations of the analogs were as follows: $84 \mathrm{mM}$ for 2 -aminoethanol, $70 \mathrm{mM}$ for 2 -aminopropanol, $62 \mathrm{mM}$ for 2-amino-1-butanol, $27 \mathrm{mM}$ for leucinol, $38 \mathrm{mM}$ for 2 aminobutane, $3 \mathrm{mM}$ for 2 -aminopentane, $21 \mathrm{mM}$ for 2 -aminohexane, $8 \mathrm{mM}$ for 2-amino-3-methylpentane, $6 \mathrm{mM}$ for 2-amino-4-methylhexane, and $2 \mathrm{mM}$ for 2-amino-6-methylheptane. Isoleucinol and valinol up to the concentration of $10^{-1} \mathrm{M}$ did not cause any growth inhibition. Very volatile compounds like 2aminoethane and 2-aminopropane were not tested in the growth experiments. 
The results showed again that 1-methyl type analogs are more efficient inhibitors than 1-hydroxymethyl type compounds.

\section{DISCUSSION}

It is supposed that in the present models of the transamination reaction mechanism the carboxyl group is needed to put the amino acid into the right position in the transaminase enzyme molecule, and thus that the group attachment on the enzyme determines which of the bonds in the Schiff base is broken in the reaction.

The results in Table 1 strongly support this evidence that the carboxyl group is essential for the enzymatic leucine-2-oxoglutarate transamination reaction. All the analogs tested were inhibitory and these analogs either had a 1-hydroxymethyl or a 1-methyl group instead of the carboxyl group in the amino compound. The change of the group resulted in inhibition with both types of analogs, although the 1-methyl group gave higher inhibition values than the 1-hydroxymethyl group. The study showed that the effect of the side chain was less strong. This is understandable if it is the carboxyl group, and not the side chain, that plays the key role in stabilizing the structure of the Schiff base or if the specificity of the leucine aminotransferase from $E$. coli is very low. Similar results were obtained with valyl-sRNA synthetase from $E$. coli when various amino acid analogs were studied. ${ }^{8}$ The carboxyl group of the amino acid seems to be essential for the liberation of hydrogen from the $\alpha$ carbon (Fig. 2). However, the $K_{\mathrm{i}}$ values in Fig. 1 show, that the binding of the analogs is of about the same order as the $K_{m}$ values for L-leucine. This finding is consistent with the results of experiments with valyl-sRNA synthetase. ${ }^{8}$ It is thus likely that the role of the carboxyl group in the binding process is very weak but may be important in the Schiff base structure, as suggested by Heinert and Martell ${ }^{9}$ and by Martell. ${ }^{10}$

Acknowledgements. The authors wish to thank Mr. P. Koponen for skillful assistance and the National Research Council for Sciences for financial aid (to R. R.). Also we thank Dr. K. Pihlaja, of the Department of Chemistry, for the NMR service.

\section{REFERENCES}

1. Ivanov, V. I. and Karpeisky, M. Ya. Advan. Enzymol. 32 (1969) 21.

2. Jenkins, W. T. In Snell, E. E., Fasella, P. M., Braunstein, A. and Rossi Fanelli, A., Eds., Chemical and Biological Aspects of Pyridoxal Catalysis, Pergamon, Oxford 1963 , p. 139 .

3. Dunathan, H. C. Proc. Natl. Acad. Sci. U.S. 55 (1966) 712.

4. Raunio, R. P. Acta Chem. Scand. 22 (1968) 2733.

5. Raunio, R. P. Acta Chem. Scand. 23 (1969) 1168.

6. Vogl, O. and Pöhm, M. Monatsh. 83 (1952) 541.

7. Dixon, M. Biochem. J. 55 (1953) 170.

8. Owen, S. L. and Bell, F. E. J. Biol. Chem. 245 (1970) 5515.

9. Heinert, D. and Martell, A. E. J. Am. Chem. Soc. 84 (1962) 3257.

10. Martell, A. E. In Snell, E. E., Fasella, P. M., Braunstein, A. and Rossi Fanelli, A., Eds., Chemical and Biological Aspects of Pyridoxal Catalysis, Pergamon, Oxford 1963, p. 26.

Received October 16, 1972.

Acta Chem. Scand. 27 (1973) No. 3 